\title{
Septic arthritis in neonates due to Extended Spectrum ß-Lactamase producing Klebsiella pneumoniae - Outbreak analysis and long term follow-up
}

\author{
Vrithmani Aprameya Indumathi', C Ranganatha ${ }^{2}$, Punith Kempegowda ${ }^{3}$ \\ 'Department of Microbiology, M S Ramaiah Medical College, MSRIT Post, Bengaluru-560054, India \\ ${ }^{2}$ Department of Orthopedics, M S Ramaiah Medical College, MSRIT Post, Bengaluru-560054, India \\ ${ }^{3}$ Ealing Hospital NHS Trust, Uxbridge Road, UB1 3HW, London, UK
}

doi: 10.3396/ijic.v8i3.028.12

\begin{abstract}
Between May and June 2002, we recorded an outbreak of Septic Arthritis in neonates due to Extended Spectrum ß-Lactamase producing Klebsiella pneumoniae at our neonatal intensive care unit. The confirmed cases were then followed up to look for long term morbidities associated with the infection. We analyzed the microbiological and epidemiological features of the outbreak and the various measures implemented to ward off the same. We also studied the long term morbidities associated with nosocomial infection.
\end{abstract}

All neonates admitted to the NICU during this period were screened and those with clinico-microbiological evidence of septic arthritis were included in the study. Microbiological screening of the environment, personnel, patients and their attendants were conducted and 85 different environmental sites were sampled. Six neonates

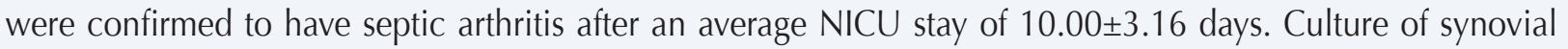
fluid grew K. pneumoniae. All isolates had similar biochemical and antibiotic resistance pattern suggestive of clonality of infection. Plasmid analysis of these samples revealed a single plasmid (35.8MD) in all the isolates. Similar K. pneumoniae strain with the same plasmid was isolated from the water samples which was stored

\author{
Corresponding Author \\ Dr Punith Kempegowda \\ Clinical Fellow Post-graduate, Level 3, Ealing Hospital NHS Trust, Uxbridge Road, Southall, UB1 3HW \\ London, UK \\ Email: drpunith@gmail.com
}


and used for hand washing. During the nine year follow up period, three neonates recovered completely, one developed dislocation of the joint and two were lost to follow up. Our study demonstrates the need for a comprehensive infection control measure and intensive monitoring of the NICUs for the ESBL producing K. pneumoniae.

\section{Key words}

Arthritis, infectious; Infant, newborn; Klebsiella pneumoniae; beta-lactamases; drug resistance, microbial

\section{Introduction}

Klebsiella pneumoniae is one of the most common organism causing nosocomial infections, ranging from bacteremia to localised systemic infection. ${ }^{1}$ Of the many alternates of antibiotic resistant $K$. pneumoniae, the Extended Spectrum B-Lactamase (ESBL) producing variant poses a major therapeutic challenge. Association of $K$. pneumoniae with nosocomial infection is not uncommon. Numerous outbreaks of K. pneumoniae in wide range of hospital settings have been reported worldwide..$^{2-5}$

Septic arthritis in infancy and children is a true clinical emergency. A delay in the treatment could result in complete destruction of articular cartilage and the underlying epiphysis, and dislocation of the joint. ${ }^{6}$ Nosocomial neonatal septic arthritis due to $K$. pneumoniae has been reported from various countries. $^{7-9}$

We witnessed an outbreak of septic arthritis in the Neonatal Intensive Care Unit (NICU) at our centre which was controlled on implementing many stringent measures. In the present report, we analyzed the microbiological, epidemiological and clinical features of the outbreak and the various measures implemented to ward off the same. We also followed up the affected case over nine year to look for the long term morbidity following the infection.

\section{Methodology \\ Identification of the Outbreak}

During our routine surveillance of the hospital reports, we noticed an increase in the number of septic arthritis in the month of May, 2002. An approval was obtained from the institution's ethical committee to investigate this unusual rise in infections. All neonates admitted to the NICU between May and June 2002 were screened.
Surveillance cultures of the gastrointestinal tracts of all these infants were performed by obtaining rectal swabs. The cultures were repeated weekly until the end of June 2002.

The diagnosis of septic arthritis was made by clinical examination, ultrasonography and radiographic evidences. Samples of synovial fluid from the affected joints and blood of the suspected cases were cultured as per standard guidelines. ${ }^{10}$

Patient demographics, medical equipment-common use and cleaning procedures, nursing staff allocations, cot placement and sharing between the neonates, common involvement in invasive procedures to the neonates and visitors were investigated in depth to identify the cause of outbreak.

Microbiological screening of the environment, personnel, patients and their attendants were conducted and 85 different environmental sites were sampled. ${ }^{11}$ Time-motion analysis of the outbreak cases was conducted by studying the case records, details of contact with health care workers, procedures conducted and equipment used. This was correlated with spatial distribution of the outbreak cases in the NICU.

\section{Microbiological assessment}

K. pneumoniae isolates were identified by $\mathrm{API} 32 \mathrm{E}$ (bioMerieux) and their antibiotic susceptibilities to different antibiotics were determined by Kirby Bauer's disc diffusion technique as per CLSI guidelines. ${ }^{12}$

The ESBL production was detected by double disc test. ${ }^{13}$ The screening for ESBL-producing organisms was done using cefpodoxime $(\leq 17 \mathrm{~mm})$, ceftazidime ( $\leq 22$ $\mathrm{mm})$, aztreonam $(\leq 27 \mathrm{~mm})$, cefotaxime $(\leq 27 \mathrm{~mm})$, and 
ceftriaxone $(\leq 25 \mathrm{~mm})$. If the organisms showed a zone of inhibition lower than the minimum for any antibiotic disc, ESBL positivity was suspected. The phenotypic confirmation was done by testing the strain against ceftazidime and ceftazidime/clavulanic acid. A >5mm diameter of the zone of inhibition for ceftazidime/ clavulanic acid in comparison to ceftazidime was considered indicative of ESBL production. Escherichia coli ATCC 25922 and K. pneumoniae 700603 were used as ESBL-negative and ESBL-positive reference strains respectively.

To determine the clonal similarity of the isolates, plasmid analysis was done by the alkaline lysis technique using the commercially available kits (CAT\#KT06, Bangalore Genei Pvt Ltd). ${ }^{14}$ All isolates were stored on Lauria Bertini agar containing $25 \mu \mathrm{g} /$ $\mathrm{ml}$ ampicillin for further studies on plasmids. The electrophoretic pattern was observed on $1 \%$ agarose gel containing ethidium bromide and the banding pattern was documented. Escherichia coli V517 was used as molecular weight marker. ${ }^{15}$ The isolated plasmids were used to transform the competent Escherichia coli $\mathrm{DH} 5 \alpha$ by treating with cold calcium chloride using the commercially available kits (CAT\#KT07 Bangalore Genei Pvt Ltd). Antibiotic susceptibilities were determined for all the transformants by the disc diffusion method as recommended by CLSI guidelines. ${ }^{16}$

\section{Infection Control measures}

During first week of May 2002, a few days prior to the onset of symptoms in the index case, there was a breakdown in the water supply to the NICU. Therefore an emergency makeshift arrangement was made by the staff to store the water in open bucket with no tap attached for washing hands. This was used by multiple users who used to dip a mug into the stored water bucket and use that for hand washing. This process continued for a period of 72 hours before it was brought to the notice of hospital infection control committee. Water shortage was then sorted out by introducing sterile water in closed containers with tap, for free flowing water; use of hand driers, and alcoholic hand rubs and sterile gloves. The nursing staffs were instructed to adhere to strict hand hygiene practices before handling the IV lines and it was done under strict supervision. This practice was continuously monitored by the person in-charge of the NICU and periodically by the infection control team. The NICU team were alerted to similar manifestations of septic arthritis/ sepsis in other babies admitted during this phase and subsequently.

Various infection control measures were also employed to limit the outbreak. The infants admitted to the NICU during this period were set in three groups - infected infants, infants exposed to the infected babies and infants who have not been exposed to either of the former groups. Separate doctors, nursing and health personnel were maintained for all three groups. Visitors to the NICU were banned during the surveillance period. However, breast feeding was continued with stringent hygienic measures. A follow-up culture of rectal swabs was done after treatment. The infants were then followed up for 3-4 days after a negative report before they were discharged.

\section{Results}

Eighty five neonates were admitted to the NICU due to various reasons during the outbreak period. Of these, six neonates were confirmed to have septic arthritis with an attack ratio of $9.2 \%$. The average age of these neonates at admission was $2.2 \pm 0.9$ days with male:female ratio being $1: 1$. The births were full term in two and premature in four. Five babies $(83.3 \%)$ were transferred from the referral centres. Two cases were admitted with bronchopneumonia, 3 other cases were admitted with hypoxic ischemic encephalopathy and the remaining one case were admitted with septicaemia secondary to necrotising enterocolitis due to K. pneumoniae. The epidemic curve (Figure 1) and timeline graph (Figure 2) summarises the various major events during the outbreak.

Septic arthritis developed after an average NICU stay of $10.00 \pm 3.16$ days. Four cases had septic arthritis of the left hip joint. One case had bilateral hip joint involvement and the other case had right elbow involvement. Culture of synovial fluid grew $K$. pneumoniae. Double disc test showed the production of ESBL in all the isolates. The isolates showed resistance to all antibiotics except cefoperazone-sulbactum, pipericillin-tazobactum, ofloxacin and imipenem. Blood cultures from two septic neonates yielded $K$. pneumoniae. All isolates had similar biochemical and 


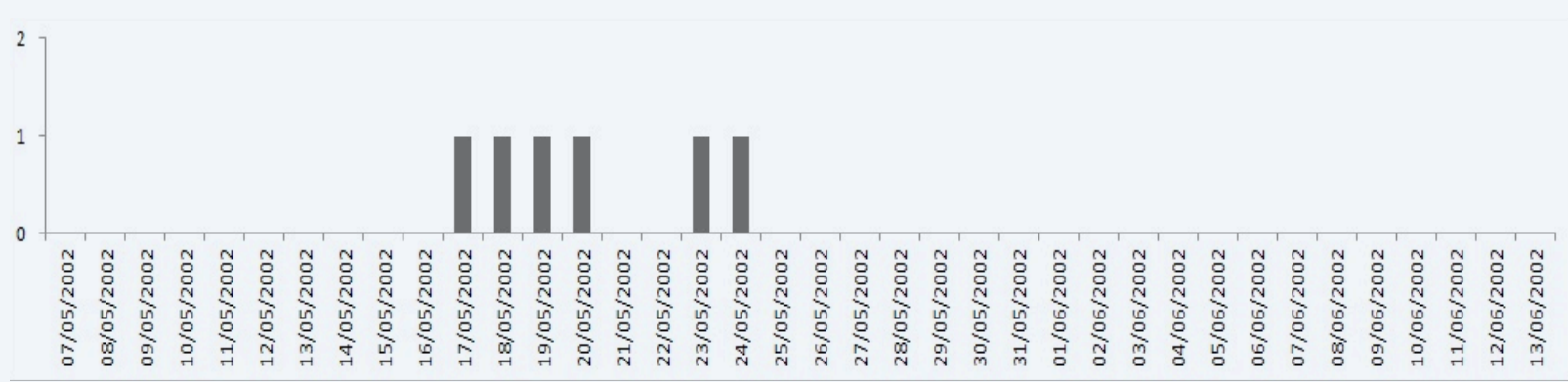

Figure 1. Epidemic curve of the outbreak of septic arthritis at our setting

antibiotic resistance pattern suggestive of clonality of infection.

Environmental screening was done to localize the source of infection and prevent further infection. There were no common staff members handling the infected babies. Also, none of the equipment used in neonatal management showed any growth on culture. The water samples from the NICU which was stored and used for hand washing showed presence of $K$. pneumoniae strain similar to the isolates from the culture. All the other samples collected from personnel and the environment was negative for $K$. pneumoniae. Five isolates from joint aspirate, one blood culture isolate and one isolate from the water were stocked for further analysis. Plasmid analysis of these samples revealed a single plasmid (35.8MD) in all the seven isolates. This plasmid could be transferred to the recipient Escherichia coli DH5 $\alpha$ and the transformants had a similar antibiotic susceptibility pattern as the clinical isolates. The transformants also showed the ESBL production as evidenced by the double disc test. The affected neonates were treated empirically with cefoperazone-sulbactum and amikacin in the high intensive care cubicle of the NICU. The same was continued for three weeks as the culture organisms showed sensitivity to these drugs. Cultures taken after 2 weeks of treatment showed no growth.

During the nine year follow up period, three neonates recovered completely, one developed dislocation of the joint and two were lost to follow up.

\section{Discussion}

ESBL producing strains of $K$. pneumoniae are considered to be nosocomial pathogens and have the highest prevalence in intensive care units. ${ }^{17-20}$ The ability of the organism to survive in the hospital niche and spread rapidly is a major concern for the treating physician. Our study elucidates an outbreak situation

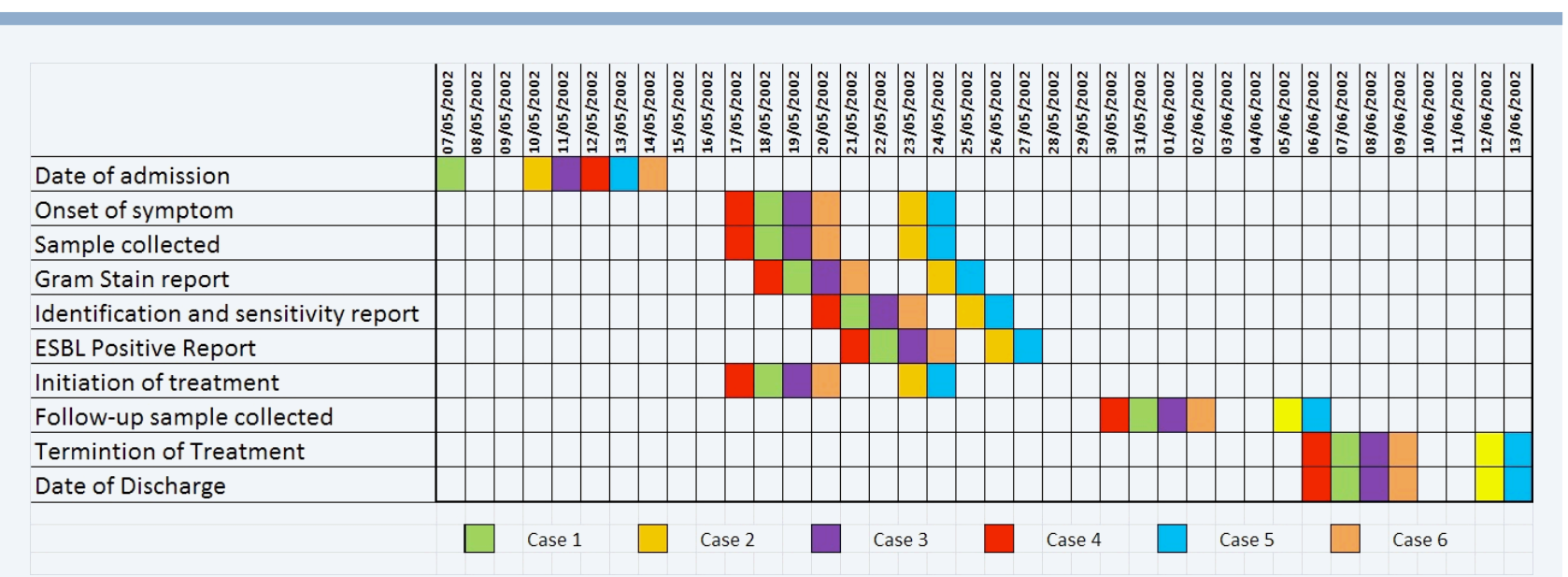

Figure 2. Time Line of the outbreak of septic arthritis at our setting: Key Information and Management process 
showing an attack rate of $9.2 \%$ occurring in a short span of two months. The isolation of $K$. pneumoniae that had identical antibiogram and plasmid bands from joint aspirates of all the neonates further supports this view. Plasmid analysis and plasmid transformation demonstrated that it was a single 35.8MD plasmid which was associated with ESBL production and multiple drug resistance.

Septic arthritis in the neonates is hematogenous in origin. The ability of $K$. pneumoniae to cause invasive infections in remote sites via blood stream is welldocumented. ${ }^{21}$ Usually infection starts in the most vascular part of the bone, i.e. metaphysis wherein there is sluggish blood flow through arterial loops which helps in establishing colonies. Then it spreads to the adjacent growth plate via transphyseal blood vessels to the epiphysis and rupture into the joint space because the metaphysis is located within the synovial membrane. ${ }^{22}$

Risk factors for the emergence of ESBL producing $K$. pneumoniae have been well demonstrated. ${ }^{17}$ This includes treatment in the ICU, prolonged hospital stay, instrumentation and antibiotic exposure to particularly the cephalosporins. ${ }^{21}$ Our study showed that each of the six cases had all of these risk factors. Our NICU had the protocol for using third generation cephalosporin, cefotaxime, as an empiric choice for treating suspected septic babies. However, there were no policy guidelines as to when the antibiotics had to be discontinued. This perhaps led to overuse of cephalosporins in the NICU which may be the reason for the ESBL Klebsiella strain in circulation. Usage of cephalosporins selects ESBL organisms. In order to stem the emergence of ESBLs, the HICC decided with the microbiology dept to closely monitor the use of cephalosporins and restrict its use to only those that truly needed treatment.

Outbreak of neonatal septic arthritis related to environmental factors has been well-documented. ${ }^{8}$ Adeyemo et al. reported an outbreak of neonatal septic arthritis by K. pneumoniae, which coincided with a period of severe water shortage which affected the hospital. ${ }^{23}$ Contamination of environmental surfaces, hands of the health care workers and transfer of patients between the hospitals have correlated with ESBL producing K. pneumoniae infections. ${ }^{17}$ In the present study, we found stored water used for hand wash was the only site that yielded ESBL $K$. pneumoniae, which was clonally identical to the clinical isolates indicating this could be the source of the outbreak. The contamination might have occurred via the hands of health care workers or the fomites.

Patient to patient transmission of $K$. pneumoniae has been documented in several studies and has been correlated with intestinal colonization. ${ }^{21}$ There is a strong probability of patient to patient spread via the hands of the health care personnel in this situation, because the cases were admitted to the high intensive care cubicle of the NICU in quick succession.

Transfer of the patients from referral hospitals and dependent centres is another risk factor for nosocomial ESBL producing K. pneumoniae infection. Our study demonstrated that five out of the six babies $(83.3 \%)$ were transferred from the referral centres. This included the index case that was septic and had necrotising enterocolitis. The ESBL producing $K$. pneumoniae might have gained entry into the NICU by this neonate. Since routine screening of the new admissions for ESBL producers is not a practice in our hospital, we could only speculate about this source.

The reported duration of the outbreak due to $K$. pneumoniae varies. ${ }^{15}$ The outbreak that occurred in our hospital could be contained within one month of the index case. Closure of the unit, cohort nursing, and implementation of alcohol based hand disinfectants, restriction of cephalosporin use and adequate measures to ensure supply of clean water helped in aborting the outbreak.

The long-term morbidity in the neonates is alarming since $16.6 \%(1 / 6)$ of our patients developed long-term sequel in the form of dislocation of the hip joint. The morbidity may be higher since two of the cases were lost for follow up.

In our setting, ESBL K. pneumoniae were not routinely isolated in the NICU and an occasional Klebsiella would be isolated from a community acquired blood stream infections, usually a non-ESBL strain. Septic arthritis was an unusual finding and all the neonates reported 
in the study manifested with similar manifestation with no preceding signs of infection.

Although other environmental sites and samples from the hands of the health care workers did not yield any Klebsiella, it must be remembered that the cultures were collected after the implementation of infection control measures and hand hygiene measures were in place, hence posing a limitation to identify the true source of infection, if any.

\section{Limitation}

Although we were able to confirm the organism phenotypically, genotypic confirmation could not be done due to limited resources and lack of technology. Also, faecal screening was not done due to financial constraints. Hence we were not able to identify any other carriers, if any.

\section{Conclusion}

The implication of water shortage leading to noncompliance and inadequate hand hygiene is a serious problem faced by many intensive care units in countries without adequate clean tap water facility. Our study demonstrates the need for a comprehensive infection control measure and intensive monitoring of the NICUs for the ESBL producing K. pneumoniae. Restricted use of third generation cephalosporins is imperative because the selective pressure of these antibiotics can lead to the emergence and spread of ESBL producing K. pneumoniae.

\section{References}

1. Rahal J. Extended spectrum ß-lactamases: how big is the problem. Clin Microbiol Infect 2000; 2: 2-6.

2. Gupta A, Della-Latta P, Todd B, et al. Outbreak of extendedspectrum beta-lactamase-producing Klebsiella pneumoniae in a neonatal intensive care unit linked to artificial nails. Infection control and hospital epidemiology 2004; 25: 210-215. http:// dx.doi.org/10.1086/502380

3. Royle J, Halasz S, Eagles G, et al. Outbreak of extended spectrum B-lactamase producing Klebsiella pneumoniae in a neonatal unit. Archives of disease in childhood Fetal and neonatal edition 1999; 80: F64. http://dx.doi.org/10.1136/ fn.80.1.F64

4. Pena C, Pujol M, Ardanuy C, et al. An outbreak of hospitalacquired Klebsiella pneumoniae bacteraemia, including strains producing extended-spectrum B-lactamase. Journal of Hospital Infection 2001; 47: 53-59.

5. Gaillot O, Maruejouls C, Abachin E, et al. Nosocomial outbreak of Klebsiella pneumoniae producing SHV-5 extendedspectrum beta-lactamase, originating from a contaminated ultrasonography coupling gel. Journal of clinical microbiology 1998; 36: 1357.
6. Frank G, Mahoney HM, Eppes SC. Musculoskeletal infections in children. Pediatric clinics of North America 2005; 52: 10831106. http://dx.doi.org/10.1016/j.pcl.2005.04.003

7. Félix V, Alonso J, Arribas M, Márquez M, Rodríguez L, Ureta A. [Neonatal septic arthritis. Our experience in 14 cases (author's transl)]. Anales españoles de pediatría 1981; 14: 305.

8. Narang A, Mukhopadhyay K, Kumar P, Bhakoo O. Bone and joint infection in neonates. Indian journal of pediatrics 1998; 65: 461-464. http://dx.doi.org/10.1007/BF02761144

9. Abuekteish F, Daoud A, Mesmar M, Obeidat A. Nosocomial neonatal septic arthritis. European journal of pediatrics 1996; 155: 102-105. http://dx.doi.org/10.1007/BF02075760

10. Forbes BA, Sahm DF, Weissfeld AS. Bailey \& Scott's diagnostic microbiology: Mosby Saint Louis; 1998.

11. Mehtar S. Hospital Infection Control: Setting Up a Costeffective Programme. Oxford: Oxford Medical Publications; 1992.

12. NCCLS. How to define and determine reference intervals in the clinical laboratory: approved guideline. NCCLS document C28-A2. Second Edition ed. Wayne, PA: NCCLS; 2000.

13. Livermore DM, Brown DFJ. Detection of ß-lactamasemediated resistance. Journal of Antimicrobial Chemotherapy 2001; 48: 59. http://dx.doi.org/10.1093/jac/48.suppl_1.59

14. Bimboim H, Doly J. A rapid alkaline extraction procedure for screening recombinant plasmid DNA. Nucleic acids research 1979; 7: 1513. http://dx.doi.org/10.1093/nar/7.6.1513

15. Macrina FL, Kopecko DJ, Jones KR, Ayers DJ, McCowen SM. A multiple plasmid-containing Escherichia coli strain: convenient source of size reference plasmid molecules. Plasmid 1978; 1: 417-420. http://dx.doi.org/10.1016/0147-619X(78)90056-2

16. NCCLS. Performance standards for antimicrobial disk susceptibility tests. Approved standard. NCCLS document M2A7. 7th edition ed. Wayne, PA: NCCLS; 2000.

17. Quale J, Bratu S, Landman D, Heddurshetti R. Molecular epidemiology and mechanisms of carbapenem resistance in Acinetobacter baumannii endemic in New York City. Clinical infectious diseases 2003; 37: 214. http://dx.doi. org/10.1086/375821

18. Rebuck JA, Olsen KM, Fey PD, Langnas AN, Rupp ME. Characterization of an outbreak due to extended-spectrum ß-lactamase-producing Klebsiella pneumoniae in a pediatric intensive care unit transplant population. Clinical infectious diseases 2000; 31: 1368. http://dx.doi.org/10.1086/317474

19. Wiener J, Quinn JP, Bradford PA, et al. Multiple antibioticresistant Klebsiella and Escherichia coli in nursing homes. JAMA: The Journal of the American Medical Association. 1999; 281: 517. http://dx.doi.org/10.1001/jama.281.6.517

20. Pałucha A, Mikiewicz B, Gniadkowski M. Diversification of Escherichia coli Expressing an SHV-Type Extended-Spectrum ß-Lactamase (ESBL) during a Hospital Outbreak: Emergence of an ESBL-Hyperproducing Strain Resistant to ExpandedSpectrum Cephalosporins. Antimicrobial agents and chemotherapy 1999; 43: 393-396.

21. Grogan J, Murphy H, Butler K. Extended-spectrum betalactamase-producing Klebsiella pneumoniae in a Dublin paediatric hospital. British journal of biomedical science 1998; 55: 111.

22. Isaacs D, Moxon ER. Neonatal infections. First Edition. Isaacs D, Moxon ER, editors. Oxford: Butterworth-Heinemann; 1991.

23. Adeyemo A, Akindele J, Omokhodion S. Klebsiella septicaemia, oseomyelitis and septic arthritis in neonates in Ibadan, Nigeria. Annals of tropical paediatrics 1993; 13: 285 289. 